\title{
Fan-out in gene regulatory networks
}

\author{
Kyung $\mathrm{H} \mathrm{Kim}^{*}$, Herbert M Sauro*
}

\begin{abstract}
Background: In synthetic biology, gene regulatory circuits are often constructed by combining smaller circuit components. Connections between components are achieved by transcription factors acting on promoters. If the individual components behave as true modules and certain module interface conditions are satisfied, the function of the composite circuits can in principle be predicted.

Results: In this paper, we investigate one of the interface conditions: fan-out. We quantify the fan-out, a concept widely used in electrical engineering, to indicate the maximum number of the downstream inputs that an upstream output transcription factor can regulate. The fan-out is shown to be closely related to retroactivity studied by Del Vecchio, et al. An efficient operational method for measuring the fan-out is proposed and shown to be applied to various types of module interfaces. The fan-out is also shown to be enhanced by self-inhibitory regulation on the output. The potential role of an inhibitory regulation is discussed.

Conclusions: The proposed estimation method for fan-out not only provides an experimentally efficient way for quantifying the level of modularity in gene regulatory circuits but also helps characterize and design module interfaces, enabling the modular construction of gene circuits.
\end{abstract}

\section{Background}

Engineering relies on modular composition, that is, the ability to combine functional units with the knowledge that the intrinsic properties of each module is unaffected to a large degree by the composition. In biology, the notion of a modular component is less clear, or at least biology has multiple definitions depending on context [1]. Here a module is defined as a self-contained functional unit whose intrinsic properties are independent of the surrounding milieu. This definition is similar to that used in engineering. For example, the intrinsic properties of a CMOS (complementary metal oxide semiconductor) NAND gate [2] is unaffected (within certain design constraints) when connected to other CMOS logic gates. That is, a NAND gate remains a NAND gate no matter what it is connected to. This property allows engineers to design, predict, and fabricate complex circuits at very low cost. The question whether such self-contained and functionally independent modules exist at the biological cellular network level is still an ongoing research problem [3]. In this paper, the design of modular synthetic components [4-10] is

\footnotetext{
*Correspondence: kkim@uw.edu; hsauro@uw.edu Department of Bioengineering, University of Washington, William H. Foege Building, Box 355061, Seattle, WA 98195-5061, USA
}

considered, and the question of modularity in natural complex systems is avoided.

In the most abstract sense, a module can be defined as follows. Given a functional unit $M$ with input $I$ and output $O$, a relation between the input and output can be defined as $O=M(I)$. Given two functional units, $M_{1}$ and $M_{2}$, where the output of $M_{1}$ serves as the input to $M_{2}$, then $M_{1}$ and $M_{2}$ are defined as modules if the relation, $\mathrm{O}_{2}=M_{2}\left(M_{1}\left(I_{1}\right)\right)$ is true. This simply means that in connecting $M_{1}$ and $M_{2}$ together, $M_{2}$ has no effect on the functional characteristics of $M_{1}$ and vice versa.

Predictable composition is of particular interest to the synthetic biology community where gene circuits are "wired" together via transcription factors (TFs) and corresponding promoters (Figure 1). This mode of wiring makes the physical construction of relatively complex networks possible $[11,12]$. However the general question of whether making a connection between two genetic units results in a predictable functional whole remains. In particular, a number of issues present themselves that include independence from the surrounding milieu (also called orthogonality [5]): domain matching and impedance bridging. The former describes the situation where the operating concentration range of an output transcription factor matches the range of the input
C Biomed Central 


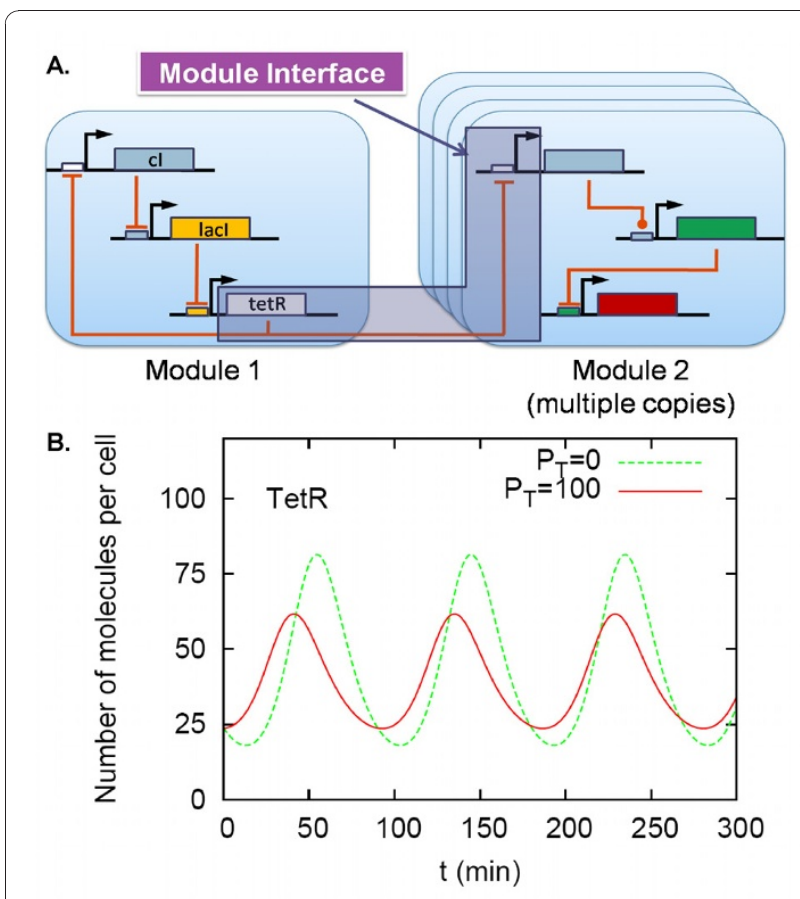

C.

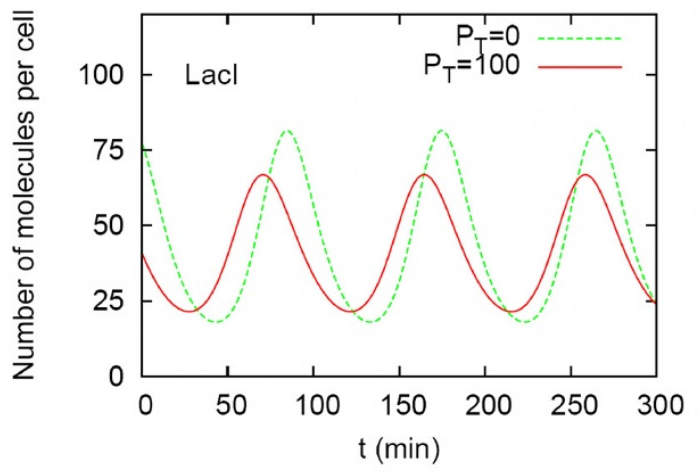

Figure 1 Gene circuit modules and their interface process (A) The repressilator (Module 1) [30] regulates multiple copies of a downstream module (Module 2). The multiple copies can be realized by placing the downstream module in a plasmid. TetR repressors (output of Module 1) regulate their specific downstreammodule promoters (input of Module 2). A module interface process (MIP) defines the collection of the processes of tetR-transcription and translation and its specific downstream-module regulation. As the number of the downstream-module promoter $\left(P_{T}\right)$ increases, the amplitude of the oscillation in the repressilator can be changed significantly ( $B$ and $C$ ). A repressilator model was obtained from the BioModels Database BIOMD0000000012 [50]. The model was modified to lower the expression levels (by changing translation efficiency to 10 and $K_{M}$ to 10 molecules per cell and the maximum transcription rate to 3 molecules per min per cell) and to add promoter binding-unbinding reactions for TetR repressors (for the detailed model description, refer to Additional File 1).

target. Impedance bridging, which is the main topic of this paper, is concerned with how much a downstream target circuit can affect the functional properties of an upstream unit. It is not to be confused with impedance matching which is related to the maximum power transfer between two circuits. There has recently been interest in defining impedance bridging in genetic and protein circuits [13-15] and a related quantity called retroactivity was introduced by Saez-Rodriguez et al. [16] and Del Vecchio et al. [17] to describe the effect of one module on another.

In electrical engineering there exist guidelines and published constraints on how many electrical modules can be driven from a source. For example, one rule of thumb for analog circuits suggests that the impedance at the input should be ten times the impedance at the driving circuit. In digital circuits, such as TTL (transistor-transistor logic) circuits [18], manufacturers will quote the fan-out and fan-in for a given electrical module. The fan-out indicates how many downstream logic gates can be connected to a given output. Exceeding these limits will potentially cause signal distortion in analog circuits and circuit failure in digital circuits. We envision the development of similar criteria for connecting two biological modules together in synthetic biology and introduce the notion of fan-out for a genetic circuit. The fan-out of a genetic circuit is defined as the maximum number of downstream promoters that can be driven from an upstream circuit signal without significant time-delay or signal attenuation.

In this manuscript, an experimental estimation method for the fan-out is proposed. This method is based on a linear relationship between a certain property of a module (response time) and the amount of load from downstream components (e.g., the number of downstream promoters in gene circuits). This linear relationship has not been discussed in previous work, for example the work by Del Vecchio et al $[17,19]$. By taking into account the linearity, we extend the retroactivity concept to the fan-out. Our analysis shows that the linear relationship holds not only for the simple module interface that Del Vecchio et al. considered [17] but also for a much wider class of interface. The linear relationship is shown to provide a unifying way for evaluating the fan-out in an efficient manner for all the interfaces belonging to the class. The fan-out can be estimated by using the autocorrelation [20-25] of gene expression noise [26-29]. During the estimation procedure the system's retroactivity can also be measured. Although our analysis is focused on genetic networks, the principles apply equally to signal transduction networks.

\section{Results and Discussion}

\section{Module interface process}

When two synthetic gene circuits are connected, transcription factors are used to connect them. The reaction processes involving the transcription factors such 
as transcription, translation, degradation, and downstream-module promoter regulation, will be called module interface processes (MIPs). For example, consider the repressilator [30]. Let us choose the TetR repressor, one of the genes comprising the oscillator, as an output of the oscillator module (Figure 1). When a downstream module has tetR-operons, the MIP includes tetR-transcription, translation, and TetR binding/unbinding to its specific operons located in the downstream module.

\section{Retroactivity and mapping between a module interface process and an RC-circuit}

We investigate a MIP by mapping it to a simple electric circuit composed of a resistor and a capacitor connected in series ( $\mathrm{RC}$ circuit). This mapping becomes significantly helpful for understanding retroactivity [17] and quantifying fan-out.

\section{Isolated case}

When an upstream output does not regulate any downstream promoter, the corresponding MIP can be modeled as a simple TF translation-degradation process (see Figure $2 \mathrm{~A}$ and $2 \mathrm{~B}$ ). The concentration of the TF, denoted by $X$, changes in time by following the equation

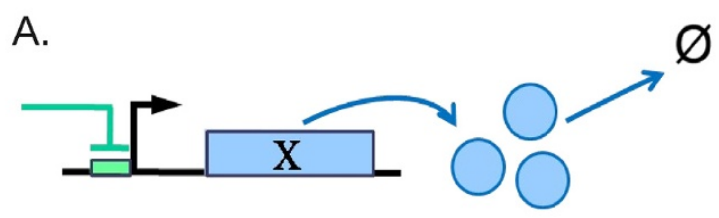

B.
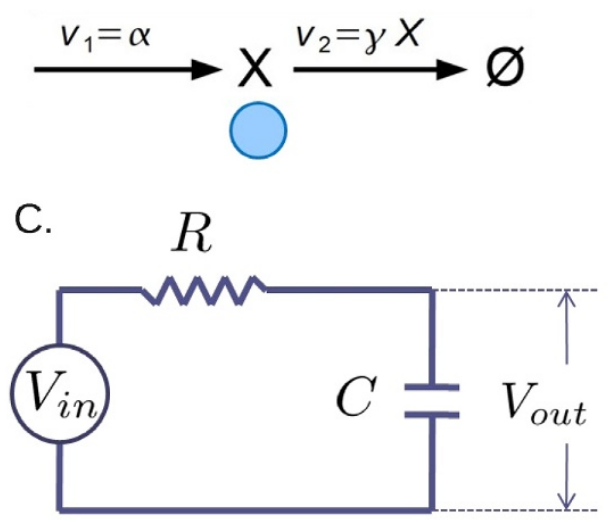

Figure 2 Isolated module output. Translation-degradation processes for $X(A)$ can be described by a simple reaction process (B) with $\alpha$ a translation rate and $\gamma X$ a degradation rate. These processes can be mapped to an $\mathrm{RC}$-circuit with $R$ resistance and $C$ capacitance by $V_{\text {out }}=X, V_{\text {in }}=\alpha / \gamma$, and $R C=1 / \gamma(C)$.

$$
\frac{d X}{d t}=\alpha(t)-\gamma X
$$

with $\alpha(t)$ the translation rate and $\gamma$ the degradation rate constant. We show how this process can be related to an RC circuit, where a resistor and capacitor are connected in series and driven by an input voltage source $V_{\text {in }}$ (Figure 2C). The total voltage drop across both the resistor and capacitor is equal to the driven voltage: $V_{\text {in }}=R I+V_{\text {out }}$, where $I$ denotes the current flowing through the resistor, and $V_{\text {out }}$ the voltage drop across the capacitor. The current is equal to the rate of charge accumulation $(Q)$ in the capacitor: $I=d Q / d t$, where the small increment $d Q$ causes the change in $V_{\text {out }}$ in proportion to $d Q: d Q=C d V_{\text {out }}$, with $C$ a proportionality constant called capacitance. Thus, the current $I$ can be expressed as $C d V_{\text {out }} / d t$. By substituting this into $V_{\text {in }}=R I+V_{\text {out }}$ and dividing the resultant equation by $R C$, we obtain

$$
\frac{d V_{\text {out }}}{d t}=\frac{V_{\text {in }}}{R C}-\frac{V_{\text {out }}}{R C},
$$

where $R C$ is known as the response time $\tau_{0}$ of the RCcircuit [31]. By comparing Eqs. (1) and (2), the following correspondence is obtained: $X=V_{\text {out }}, \alpha=V_{\text {in }} / R C$, and $\gamma=1 / R C$, and the response time is expressed as

$$
\tau_{0}=R C=\frac{1}{\gamma} .
$$

Thus, the TF-translation-degradation process (Figure 2B) can be directly mapped to the RC-circuit (Figure 2C).

\section{Connected case}

When two modules are connected (see Figure 3A), Del Vecchio et al. [17] have shown that the interfacial dynamics slows down in response to interactions with downstream components. The degree of the slowdown defines the retroactivity as follows: The retroactivity describes a number between zero and one with one being the least desirable, i.e. the interfacial dynamics are affected most. In their analysis, they assumed that the binding-unbinding process of the TF is fast enough that the process can be approximated to be in the quasi-steady state $\left(k_{o n} X+k_{\text {off }} \gg \gamma\right.$; cf. [32-34]). They also assumed that the lifetime of the bound TF is much longer than that of the unbound TFs.

Specifically, they showed that the free TF concentration $X$ changes in time by the following equation [17]

$$
\frac{d X}{d t}=(1-\mathcal{R}(X))(\alpha-\gamma X)
$$



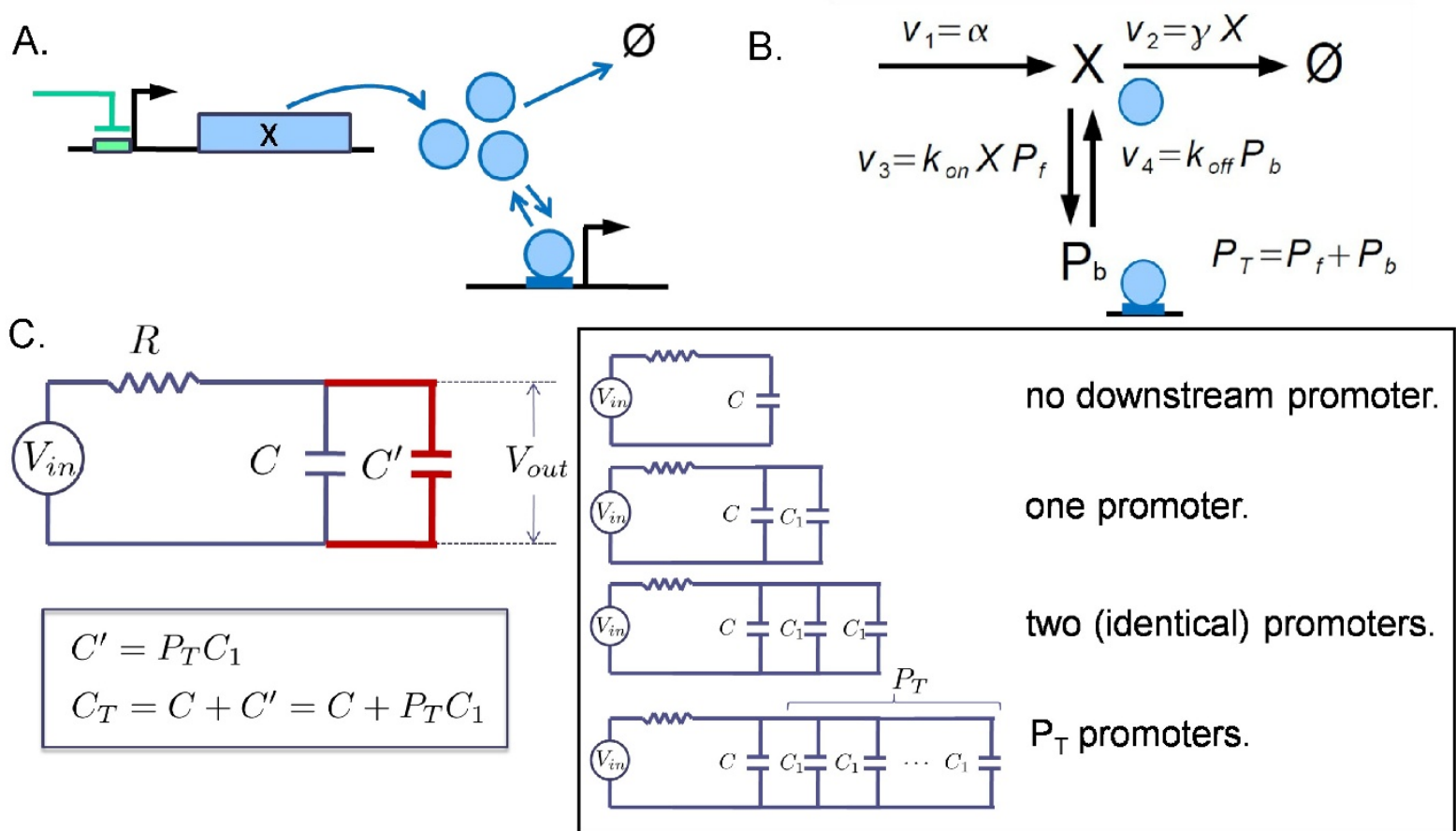

Figure 3 Module interface process. The output $X$ of an upstream module regulates the downstream-module $X$-specific promoter (A). The translation-degradation processes for $X$ and its promoter regulation can be modeled as the reaction process shown in $\mathrm{B}$, where $P_{f}, P_{b}$, and $P_{T}$ denote the numbers of free, bound, and total promoters, respectively. The reaction process is mapped to an RC-circuit with an increased capacitance by $C^{\prime}$, which is shown to be proportional to $P_{T}: C^{\prime}=P_{T} C_{1}$ with $C_{1}$ a proportionality constant. This means that each promoter acts as a capacitor with a unit load of capacitance, $C_{1}$. The total capacitance $C_{T}$ becomes the sum of the capacitance $C$ in the isolated case and the extra capacitance $C^{\prime}$.

where $\mathscr{R}(X)$ is the retroactivity, given as

$$
\mathcal{R}(X)=\left[1+\left(1+\frac{X}{K_{d}}\right)^{2} \frac{K_{d}}{P_{T}}\right]^{-1},
$$

with $K_{d}$ the dissociation constant for the TF with respect to the promoter, and $P_{T}$ the total number of the promoters. They showed that $\mathscr{R}$ is always less than 1 and non-negative. The extra factor 1 - $\mathscr{R}$ appears when compared with the isolated case, resulting in the slowdown of the dynamics. More precisely, the slow-down is due to the decrease in the factor placed in front of $X$ in Eq. (4): $\gamma(1-\mathscr{R})$, which is related to the apparent response time:

$$
\tau_{a} \equiv \frac{1}{(1-\mathcal{R}) \gamma}
$$

We consider the MIP shown in Figure $3 \mathrm{~A}$ and $3 \mathrm{~B}$ under the same assumptions as given by Del Vecchio et al [17]. To understand the retroactivity by using an RCcircuit analogy, consider a circuit shown in Figure 3C. The total capacitance becomes the sum of the two capacitances: $C_{T}=C+C$. Thus, the response time becomes $R C_{T}: \tau=R C_{T}$. The change in the output voltage is governed by the same equation as in the isolated case except the capacitance $C$ is replaced to $C_{T}$ :

$$
\frac{d V_{\text {out }}}{d t}=\frac{V_{\text {in }}}{R C_{T}}-\frac{V_{\text {out }}}{R C_{T}}=\left[1-\frac{C^{\prime}}{C+C^{\prime}}\right]\left[\frac{V_{\text {in }}}{R C}-\frac{V_{\text {out }}}{R C}\right](7)
$$

By comparing Eqs. (4) and (7), the retroactivity is given by the relative ratio of the new capacitance:

$$
\mathcal{R}=\frac{C^{\prime}}{C+C^{\prime}}
$$

(6)):

$$
\tau=R C_{T}=\frac{1}{(1-\mathcal{R}) \gamma} .
$$

Connecting downstream promoters in the MIP is therefore shown to be equivalent to connecting extra capacitors in parallel with an existing capacitor in the $\mathrm{RC}$-circuit. Due to these extra capacitors, the circuit 
takes a longer time to fully charge all the capacitors, resulting in the slow-down in the circuit response time. Biologically, the bound promoters act as a reservoir of potentially free TFs: Whenever there is a change in the number of the free TFs, the reservoir quickly buffers the change in the number of free TFs [35]. Such buffering causes transient dynamics at the interface to slow down.

\section{Response time vs. number of promoters}

The response time is shown to increase with $P_{T}$ (see the Methods section) as

$$
\tau_{P_{T}}=R\left(C+P_{T} C_{1}\right)
$$

where $C_{1}$ is a proportionality constant satisfying $C / C_{1}=$ $K_{d}\left(1+X / K_{d}\right)^{2}$. The above equation (10) can be viewed as each individual promoter contributing an extra capacitance $C_{1}$ to the total capacitance (Figure $3 \mathrm{C}$ ): $C_{T}=C+$ $P_{T} C_{1}$. The capacitance $C_{1}$ of each extra capacitor is related to a unit load onto the upstream output dynamics from a single downstream promoter. This is an interesting result and becomes useful for proposing an experimental method for estimating the fan-out.

The linear relationship between the extra capacitance and $P_{T}$ (see Figure $3 \mathrm{C}$ box) does not come from any linearization approximation, but from the fact that each downstream promoter affects the upstream as an independent effector (reservoir or sequestrator), although the sequestration itself is represented by a nonlinear reaction.

This linearity does not appear clearly in the retroactivity measure (Eq. (5)) proposed in [17], obscuring the connection to the concept of fan-out. The following section makes this connection and an efficient method for estimating the fan-out will be proposed.

\section{Gene circuit fan-out}

A gene circuit fan-out is defined by the maximum number of promoters in a downstream module that the output (transcription factor) of an upstream module can regulate without altering the output dynamics significantly. To exemplify how much the upstream module can be affected, a repressilator [30] is considered as a module and its Tet repressors as a module output (Figure $1 \mathrm{~A}$ ). When the output regulates tet $R$ promoters located in a downstream module, the oscillation amplitude of the tet $R$ expression level can be significantly changed, e.g., $40 \%$ decrease when the number of the promoter $\left(P_{T}\right)$ is changed from 0 to 100 (Figure 1B and $1 C)$. Our interest is here to quantify the maximum number of the promoters (fan-out) that the upstream module can tolerate.

Let us quantify the fan-out by considering again the simple MIP shown in Figure 3A. We consider a frequency response between the input and output voltage, $V_{\text {in }}$ and $V_{\text {out }}$, respectively (Figure $4 \mathrm{~A}$ ). In the RC circuit, the capacitor acts as a low pass filter: The gain of the signal (the ratio of the oscillation amplitude of $V_{\text {out }}$ to that of $V_{\text {in }}$ ) is at the maximum level for low frequencies and drops significantly when the circuit no longer responds as fast as the input signal changes (Figure 4B). The frequency when this happens is called the cut-off frequency $\left(\omega_{c}\right)$ (Figure $4 \mathrm{~B}$ ) and corresponds to the inverse of the response time: $1 / R C_{T}$ [31]. The cut-off frequency corresponds to the bandwidth in the low-pass filter [31], which defines the range of frequency where a signal gain is sufficiently large.

If the upstream module functions as a synthetic oscillator, there will be a practical upper limit $\left(\omega_{\max }\right)$ in the oscillator's frequency (e.g., for the repressilator, $\omega_{\max }$ can be the inverse of the repressor lifetime $=\log (2) / 10 \mathrm{~min}^{-1}$ $\sim 4$ hour $^{-1}$ [30]). If $\omega_{\max }$ is smaller than the cut-off frequency $\omega_{c}$, the oscillator output will operate in a predictable manner and the output signal will be passed downstream without any significant signal loss. As the number of the downstream promoters increases, the total capacitance increases as shown in Eq. (10) and the cut-off frequency $\left(\omega_{c}=1 / R C_{T}\right)$ decreases. For the cut-off frequency to be larger than the maximum operational frequency $\omega_{\max }$, the total number of the promoter must be smaller than a certain value, which will be called the fanout. The fan-out denoted by $F_{\omega_{\max }}$ is obtained where $\omega_{c}$

$$
\begin{aligned}
& \text { equals } \omega_{\max } \text {, i.e., } \omega_{c}=\left[R\left(C+P_{T} C_{1}\right)\right]^{-1}=\omega_{\max } \text { : } \\
& F_{\omega_{\max }}=\frac{C}{C_{1}}\left[\frac{1 / \tau_{0}}{\omega_{\max }}-1\right] .
\end{aligned}
$$

In the fan-out equation (11), there are two unknown parameters: $C / C_{1}$, and $\tau_{0}$. These can be experimentally estimated by performing two independent experiments with and without any downstream module. In each experiment, the corresponding response time, $\tau_{0}$ or $\tau_{P_{T}}$, can be estimated (by using gene expression noise as will be presented later in the Results section). Thus, one of the unknowns $\tau_{0}$ can be estimated. How can the other unknown $C / C_{1}$ be estimated from $\tau_{P_{T}}$ ? If the copy number of the promoters $P_{T}$ is known a priori, the value of $C / C_{1}$ can be obtained from Eq. (10). If the promoters are placed on plasmids, the copy number of the plasmids can be estimated depending on what type of origin of replication is used, and thus the copy number of the promoters $P_{T}$ can be known. By calculating $\tau_{0}=R C_{1}$, the other unknown, $C / C_{1}$, can be obtained. 

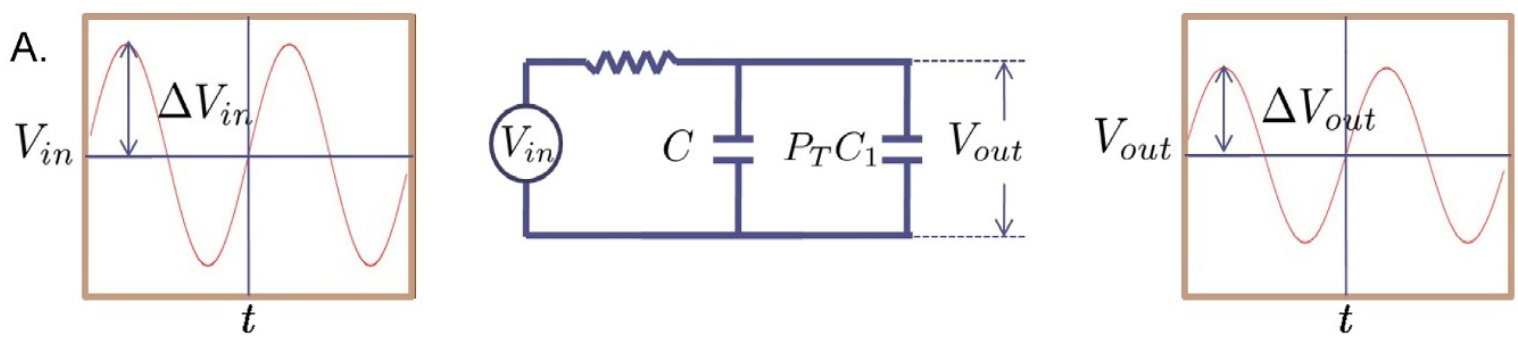

B.

\section{Desired Operating Frequency Range}

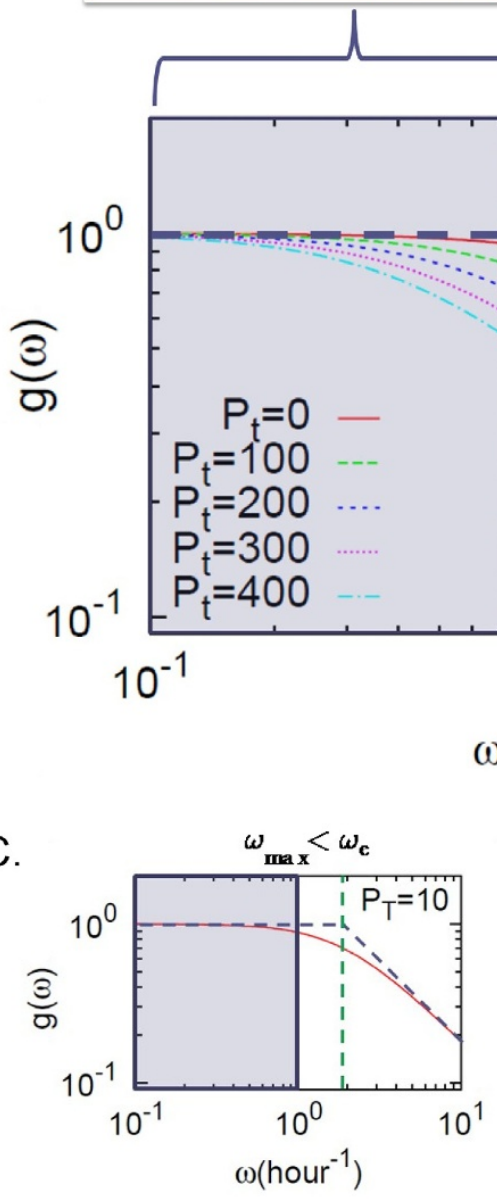

Good

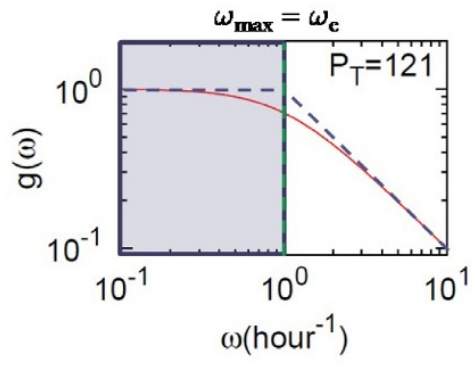

Fan-out $=121$

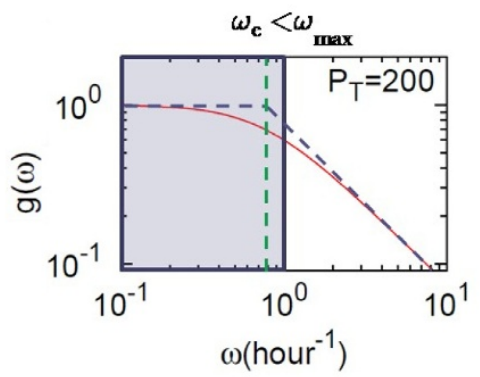

Bad

Figure 4 Frequency response of the module interface process shown in Fig. 3A. An oscillatory signal is applied at $V_{\text {in }}$ with different frequencjes (A). The signal gain is defined by the ratio of the oscillation amplitude of the output signal $\left(V_{\text {out }}\right)$ to that of the input (Vin): $g(\omega)=\frac{\Delta V_{\text {out }}(\omega)}{\Delta V_{\text {i }}(\omega)}$, and can be well approximated by $g(\omega)=\sqrt{1+\omega^{2} /\left(R C_{T}\right)^{2}}$ [31] with $C_{T}$ the total capacitance. As the number of the promoters that the output (TF) signal drives (regulates) increases, the cut-off frequency $\left(\omega_{c}=1 / R C_{T}\right)$ decreases (B). The output signal is desired to be operated within a certain frequency range, e.g., between 0 and 1 hour $^{-1}$. Here the maximum operating frequency $\omega_{\max }$ is 1 hour ${ }^{-1}$. When the cut-off frequency matches the maximum operating frequency, the corresponding number of the promoters is defined as the fan-out (C). Parameters of the model: $K_{d}=1 \mathrm{nM}\left[k_{\text {on }}=10(1 / \mathrm{nM} /\right.$ hour $), k_{\text {off }}=10(1 /$ hour $\left.)\right], \gamma=2(1 /$ hour), $\alpha=20$ (nM/hour). Here the volume of a host cell is assumed roughly equal to $1 \mu \mathrm{m}^{3}$. Under the assumption, a copy number of one corresponds to $1 \mathrm{nM}$. 


\section{Gene circuit fan-out in more general interfaces}

Up to now we have considered a simple MIP without feedback and where the degradation rate is assumed to be first-order. Here the more general case is considered and it is shown that the same or a similar fan-out function as Eq. (11) is obtained.

\section{Oligomer under directed degradation and self-regulation}

Consider that a TF, composed of $n$ monomers, is tagged for degradation and that its transcription is self-regulated as shown in Figure 5A. The fan-out function is obtained by the same equation (11), where $\tau_{0}$ is the time constant in the isolated case, given by the difference between the unscaled elasticities [36]: $1 / \tau_{0}=\varepsilon_{2}-\varepsilon_{1}$, where $\varepsilon_{1} \equiv \partial v_{1}(x, \alpha) / \partial x$ and $\varepsilon_{2} \equiv \partial v_{2}(x) / \partial x$ (refer to the Methods section). This means that the fan-out can be estimated exactly in the same way as in the monomer case as shown in Figure 3A. All the above results apply for the case that the intermediate reaction steps of the oligomerization and directed degradation are taken into account (refer to Additional File 1 and the Example 2).

\section{Multiple promoters having different affinities}

Consider the case that two different types of TF-specific promoter plasmids, having different affinities for the TF and different strength of the origin of replication. It is shown that the MIP can be mapped to an RC-circuit having two different capacitances connected in parallel to $C$ as shown in Figure 5B (see the Methods section). The fan-out of each promoter is shown to satisfy the following functional relationship between $F_{1}$ and $F_{2}$ (refer to the Methods section):

$$
1 / \omega_{\max }=\tau_{0}\left(1+F_{1} \frac{C_{1}}{C}+F_{2} \frac{C_{2}}{C}\right),
$$

where $F_{i}$ is the fan-out for promoter plasmids of the $i$-th kind, and $C_{i}$ denotes the corresponding capacitance per plasmid. If there are $N$ different kinds of promoter plasmids, all the $N$ capacitances need to be summed up in the above equation. Here the fan-out is not a single number but is given by a functional relationship between $F_{i}{ }^{\prime}$ s: The number of plasmids of different kinds needs to be balanced depending on its unit load on the retroactivity, i.e., $C_{i} / C$.

To obtain the fan-out function, it is necessary to find three unknown parameters: $\tau_{0}, C_{1} / C$, and $C_{2} / C . \tau_{0}$ can be estimated in the isolated case. $C_{i} / C$ can be estimated in the case that only the $i$-th kind of promoter plasmids exists (under the assumption that the strength of each origin of replication is already known). These three independent experiments will suffice for estimating all the unknown parameters and proposing the fan-out function Eq. (12).

\section{Multiple operators}

Consider the case that the promoter region includes multiple operators specific to an output TF (e.g., $\mathrm{O}_{1}, \mathrm{O}_{2}$, and $\mathrm{O}_{3}$ ) having different affinities (Figure 5C). Regardless the number of the operators, the same fan-out function as Eq. (11) is obtained (refer to Additional File 1).

\section{Two output signals}

When two output TFs ( $X$ and $Z$ ) regulate a downstream promoter independently, i.e., if there is no overlap between the operator regions and somehow $X$ does not interfere with the operator region of $Z$ and vice versa, the fan-out corresponding to each output TF can be obtained.

The fan-out functions like Eqs. (11) and (12) have been shown for each of the individual cases given above. For all the combinations of these individual cases the same fan-out functions will apply as well.

\section{Design scheme for fan-out enhancement}

How can we increase the fan-out? Based on the fan-out equations (11), there are two ways: increasing $C / C_{1}$ or $1 / \tau_{0}$. The way to increase the latter is to apply a negative feedback on the translation of $X$ (making $\varepsilon_{1}$ negative for the case shown in Figure $5 \mathrm{~A}$, where $1 / \tau_{0}=\varepsilon_{2}-\varepsilon_{1}$ ) and a positive feed-forward on the degradation rate (increasing $\left.\varepsilon_{2}\right)$. These applications push the cut-off frequency/bandwidth $\left(1 / \tau_{0}\right)$ further away from the maximum desired operating frequency $\left(\omega_{\max }\right)$, and enhances the fan-out as illustrated in Figure 6. A simulation study of enhancing fan-out will be presented later in this manuscript. Since the enhanced degradation and negative feedback decrease the concentration level of $X$, to prevent this, it is desirable to amplify the translation rate (which makes $\varepsilon_{1}$ more negative).

This mechanism for enhancing fan-out is exactly the one proposed by Del Vecchio et al. [17] to reduce retroactivity; when the retroactivity is small, the upstream output dynamics does not slow down significantly by connecting the output to the downstream module, meaning that the load from downstream to the upstream is small enough that many replicates of the load can be applied to the upstream without slowing down the output dynamics significantly.

One of the mechanisms, inhibitory auto-regulation, is frequently found in Escherichia coli transcription factors regulating a set of operons, e.g., for amino-acid biosynthesis where a single TF may control multiple targets, likewise for flagella formation [37]. Such motifs are called single-input-module motifs [37].

The concept of fan-out is not limited to gene regulatory circuits. In principle, as long as the same class of interface processes are found regardless of the type of biological systems, the fan-out and retroactivity concepts can be applied [1,17]. For example, in the eukaryotic MAPK pathway, doubly phosphorylated MAPK can activate a number of downstream proteins and transcription factors in the nucleus. This MAPK regulation can be 
described by the module interface process similar to the one shown in Figure 5B (in this case, many promoter plasmids instead of the two). In the MAPK pathway, there is a negative feedback from MAPK to the phosphorylation of MAPKKK [13-15]. In a recent paper by
Yu et al. [38] which showed experimentally that the related system in Yeast involving Fus3 as the negative feedback component showed linearity between receptor occupancy and downstream response in the presence of feedback. Although they did not show increased fan-out

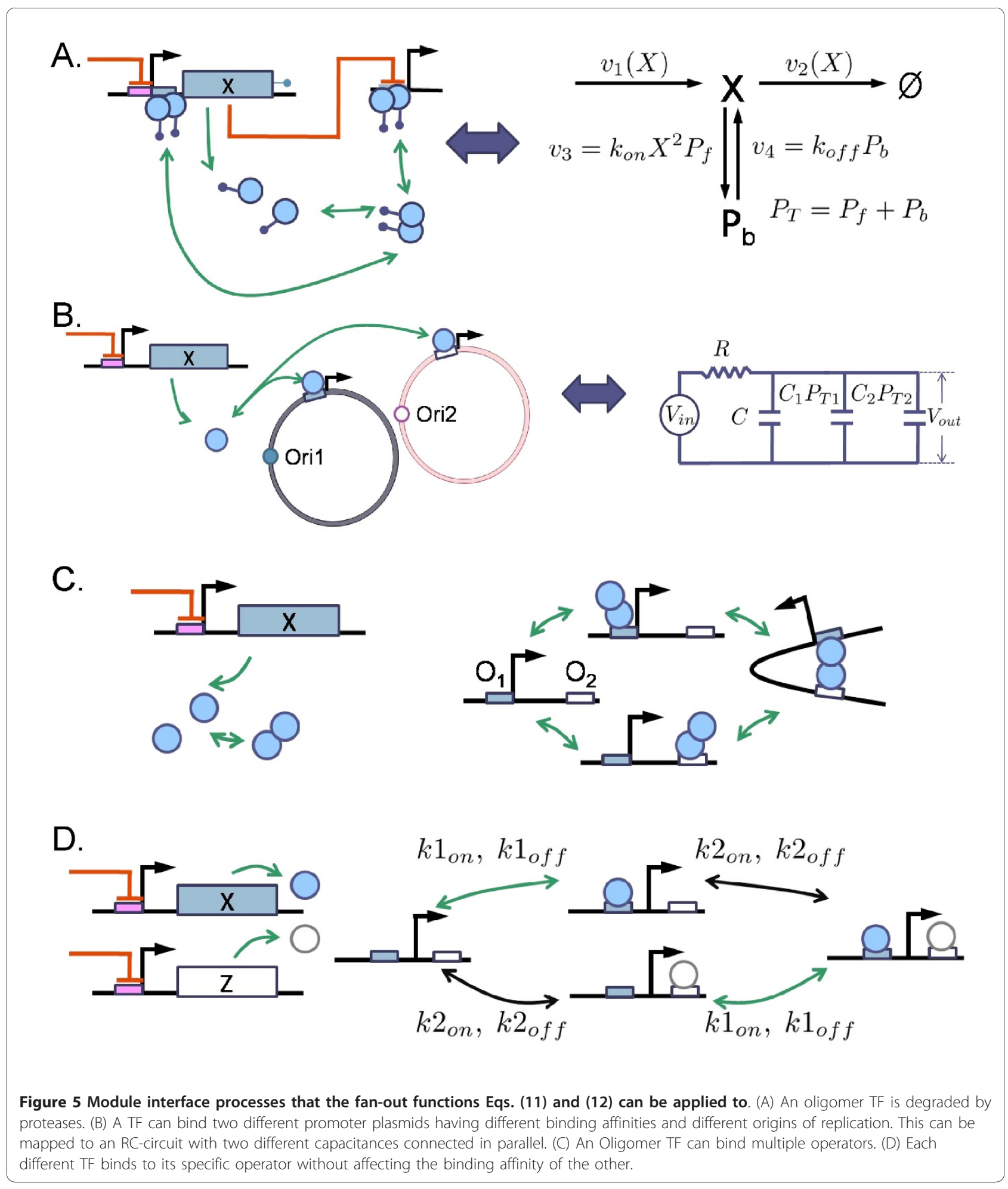


per se, the presence of linearity may suggest there is an increase in fan-out of the MAPK module, thereby permitting MAPK to effectively regulate multiple targets and multiple homologous binding sites.

\section{How to measure the time constant $\tau$}

It is known that transcription factors show significant stochastic fluctuations [22-25,39-42] (for review articles, [26-29]). Their correlation times have been measured by obtaining autocorrelations by in vivo time-lapse microscopy [22-25]. Recent numerical studies show that the correlation time is approximately equal to the response time of the deterministic case [43] and that it changes as a result of connecting two genetic systems $[19,43]$. Therefore, from the change in the correlation time, the fan-out can be estimated by using Eq. (11) as well as the

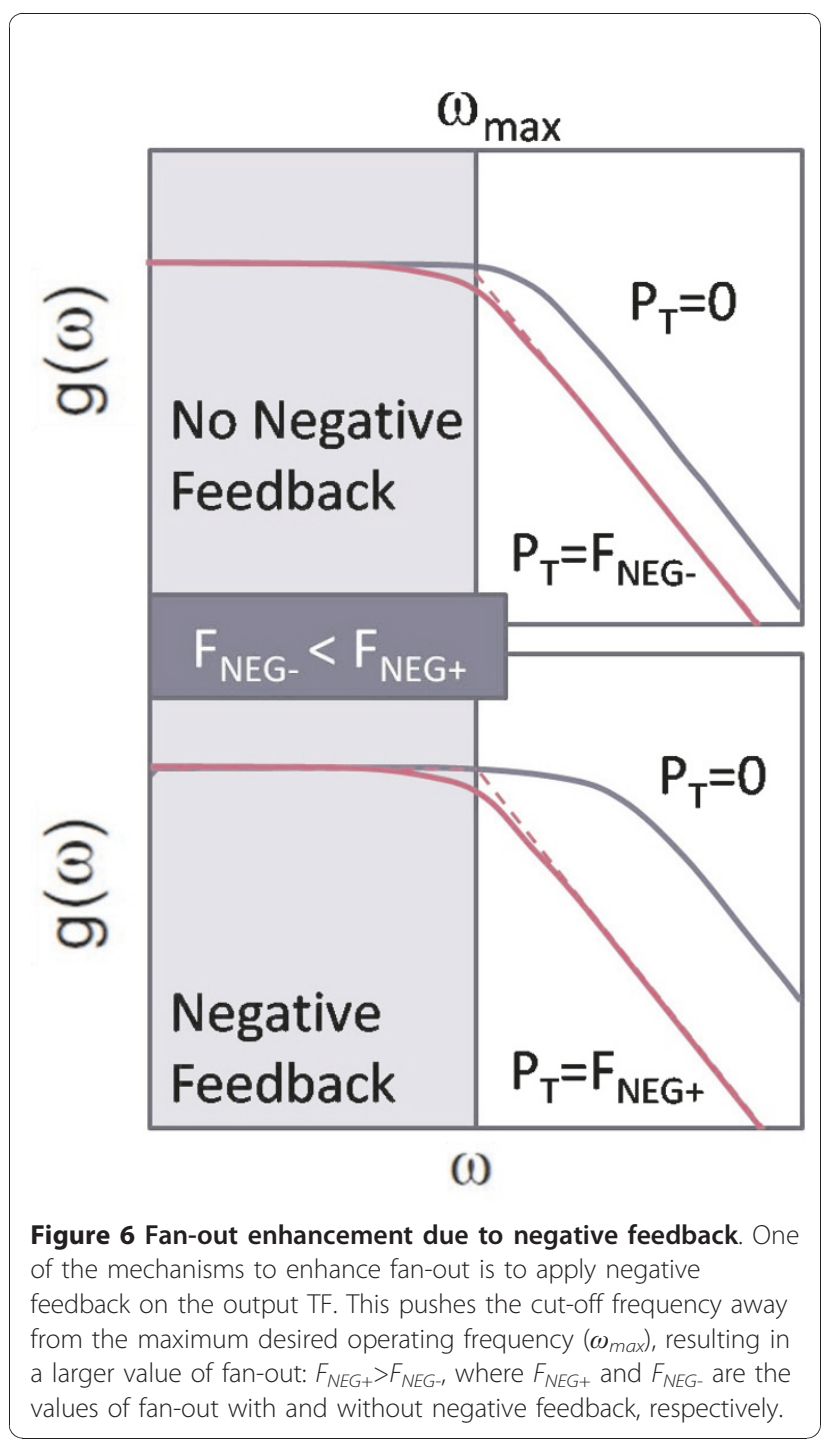

retroactivity by using $\mathcal{R}=\frac{\tau_{P_{T}}-\tau_{0}}{\tau_{P_{T}}}$ (obtained from Eq. (8) by using Eqs. (3) and (9)).

\section{Example 1: Fan-out/retroactivity estimation}

In this example, the simple MIP shown in Figure $3 \mathrm{~A}$ is considered as a model for TFs in E. coli. The average copy number of plasmids containing the specific promoters is assumed to range from 1 to 100 . The volume of $E$. coli is assumed to be roughly equal to $1 \mu \mathrm{m}^{3}$, and a copy number of one corresponds to $1 \mathrm{nM}$. As a result, the unit of $\mathrm{nM}$ is henceforth interchanged with that of copy number. A simulation using the standard Gillespie method [44] was performed (see Figure 7) and the observed autocorrelation was fitted to an exponential function: $G(\Delta t)=A \exp (-\Delta t / \tau)$ with $\tau$ a correlation time (a linear fit is conducted in the log-scale in the $y$-axis and the normal scale in the $x$-axis) and $1 / \tau$ obtained from the fitted slope (see Figure 7).

For experimentally reasonable parameter values, i.e., $\alpha$ $=20 \mathrm{nM}_{\text {hour }}{ }^{-1}, \gamma=2$ hour $^{-1}, k_{\text {on }}=10 \mathrm{nM}^{-1}$ hour $^{-1}$, and $k_{\text {off }}=10$ hour $^{-1}$, stochastic simulations were performed with and without any downstream-module promoter $\left(P_{T}\right.$ $=100$ and 0 ). The concentration levels of the total TF was recorded for 48 hours (corresponding to experimental time) with frequency 50 times per hour, the autocorrelation of this signal was fitted to an exponential function, and the response time measured (see Figure 7) [43]. The error bar of the time constant was obtained from 10 independent replicates of the autocorrelation.

When the translation rate was set to $20 \mathrm{nM}$ hour $^{-1}, \tau_{0}$ and $\tau_{100}$ were obtained to be $0.52 \pm 0.06$ hour and $0.9 \pm$ 0.1 hour, respectively. The value of $C / C_{1}$ was obtained to be $140 \pm 20$, by using

$$
\frac{C}{C_{1}}=P_{T} \frac{R C}{R C_{T}-R C}=\left.P_{T} \frac{\tau_{0}}{\tau_{P_{T}}-\tau_{0}}\right|_{P_{T}=100},
$$

where $C_{T}-C=P_{T} C_{1}$ was used. From Eq. (11), the fan-out function for this MIP was obtained:

$$
F_{\omega_{\max }}=140[ \pm 20]\left(\frac{1 / 0.9[ \pm 0.1]}{\omega_{\max }}-1\right) .
$$

If the upstream module is a synthetic oscillator with a maximum operating frequency $\omega_{\max }=1$ hour $^{-1}$, the fanout becomes $F=130 \pm 20$. This means that promoter plasmids with low, medium, and high copy numbers can be used without affecting the TF dynamics, if a single TF-specific operator site resides on a plasmid. The retroactivity can also be estimated from the measured values of $\tau_{0}$ and $\tau_{100}: \mathscr{R}=0.4 \pm 0.1$. 


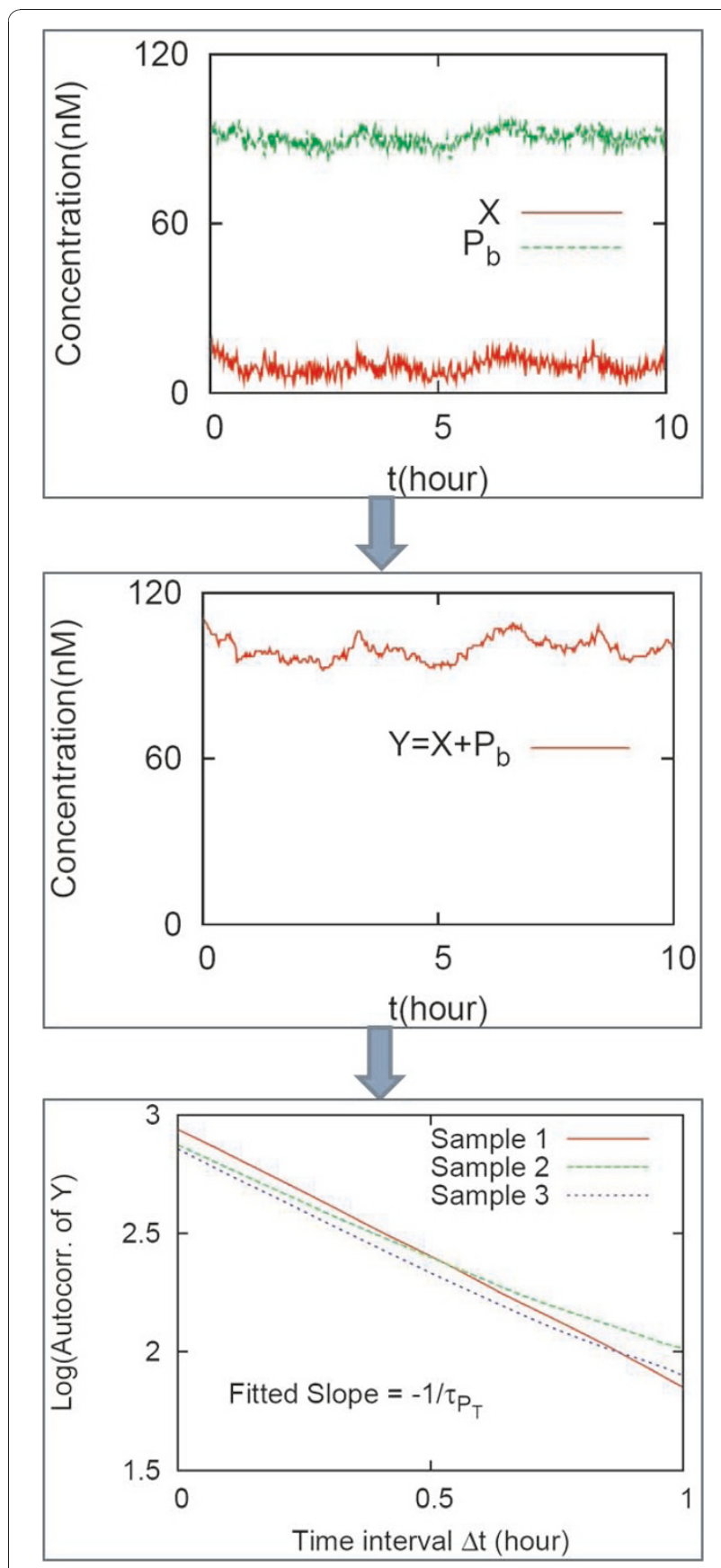

Figure 7 Estimation of response time constants from autocorrelation functions. The concentrations of free and bound TFs ( $X$ and $P_{b}$ respectively) fluctuate stochastically for the MIP shown in Fig. 3A. The total TF concentration $\left(X+P_{b}\right)$ can be observed experimentally; for example, when the TF is tagged for fluorescence, the fluorescence intensity could reflect the total TF concentration [43]. The autocorrelation of the total is fitted to an exponential function: A linear fit is taken in the semi-log scale. The inverse of the fitted slope corresponds to the response time constant $\tau_{P_{T}}$. The error bar of the $\tau_{P_{T}}$ are estimated from 10 independent replicates of autocorrelation functions (only three samples are shown in the figure). The same parameter values are used as in the Fig. 4 caption and the value of $P_{T}$ is set to 100 .
If the translation rate is reduced by half (now, $\alpha=10$ $\mathrm{nM}$ hour $^{-1}$ ), the free TF concentration decreases by half. As the concentration decreases, the retroactivity increases $[17,43]$ and the fan-out decreases. The values of $\tau_{0}$ and $\tau_{100}$ are obtained to be $0.52 \pm 0.07$ hour and $=$ $1.75 \pm 0.04$ hour, respectively. For the same $\omega_{\max }=1$ hour $^{-1}$, the fan-out becomes $F=40 \pm 1$. This would mean that only low copy number plasmids can be safely used. The retroactivity is estimated to be $0.70 \pm 0.05$.

\section{Example 2: Fan-out enhancement by applying negative feedback}

In this example, the MIP of dimer TFs is considered that are under inhibitory self-regulation as shown in Figure $5 \mathrm{~A}$. The reaction process can be described by the following set of reactions:

$$
\begin{aligned}
& \stackrel{\frac{\alpha}{1+\beta X_{2}}}{\longrightarrow} X \stackrel{\gamma X}{\longrightarrow} \varnothing \\
& X+X \underset{k_{2} X_{2}}{\stackrel{k_{1} X(X-1)}{\rightleftharpoons}} X_{2} \stackrel{\gamma_{2} X_{2}}{\rightleftharpoons} \varnothing \\
& X_{2}+P_{f} \frac{k_{o n} X_{2} P_{f}}{k_{o f f} P_{b}} P_{b}
\end{aligned}
$$

where $\beta$ is introduced to turn on and off the negative feedback. The following parameter values are used: $\alpha=$ 20(nM/hour), $\gamma=2\left(1 /\right.$ hour), $k_{1}=20\left(1 / \mathrm{nM} /\right.$ hour), $k_{2}=1$ (1/hour), $\gamma_{2}=2(1 /$ hour $), k_{\text {on }}=10(1 / \mathrm{nM} /$ hour $)$, and $k_{\text {off }}=$ $10(1 /$ hour $)$ for the case without any feedback $(\beta=0)$. For the case with negative feedback $(\beta=0.25)$, the value of $\alpha$ was adjusted to match the same expression level of $X$ as the case without feedback: $\alpha=43$. The data are sampled in the identical way as described in the Example 1 and the response time constants were measured from the autocorrelations of the total TF concentrations $\left(X+2 X_{2}+2 P_{b}\right)$.

Figure 8A shows that $\tau_{P_{T}}$ estimated from the autocorrelations linearly increases with $P_{T}$ while satisfying the deterministic prediction based on Eq. (10), which was computed by using Mathematica [45] (its notebook file is provided in Additional File 2). The value of $C / C_{1}$ was estimated from both the slope $\left(R C_{1}\right)$ and y-intercept $\left(\tau_{0}=R C\right)$ of the graph shown in Figure 8A: $R C_{1}=0.016$ \pm 0.002 hour and $\tau_{0}=0.49 \pm 0.04$ hour for the case without feedback. For $\omega_{\max }=1$ hour $^{-1}$, the fan-out $F_{N e g-}$ was estimated to be $32 \pm 7$. For the case with feedback, the values of $R C_{1}$ and $R C$ were obtained to be $0.009 \pm$ 0.001 hour and $0.31 \pm 0.05$ hour, respectively. The fanout $F_{\text {Neg+ }}$ was estimated to be $77 \pm 15$. With negative feedback, the fan-out was increased two-fold.

This fan-out enhancement can be understood in terms of the cut-off frequency increase. The signal gain in the output signal (total transcription factor) with respect to 

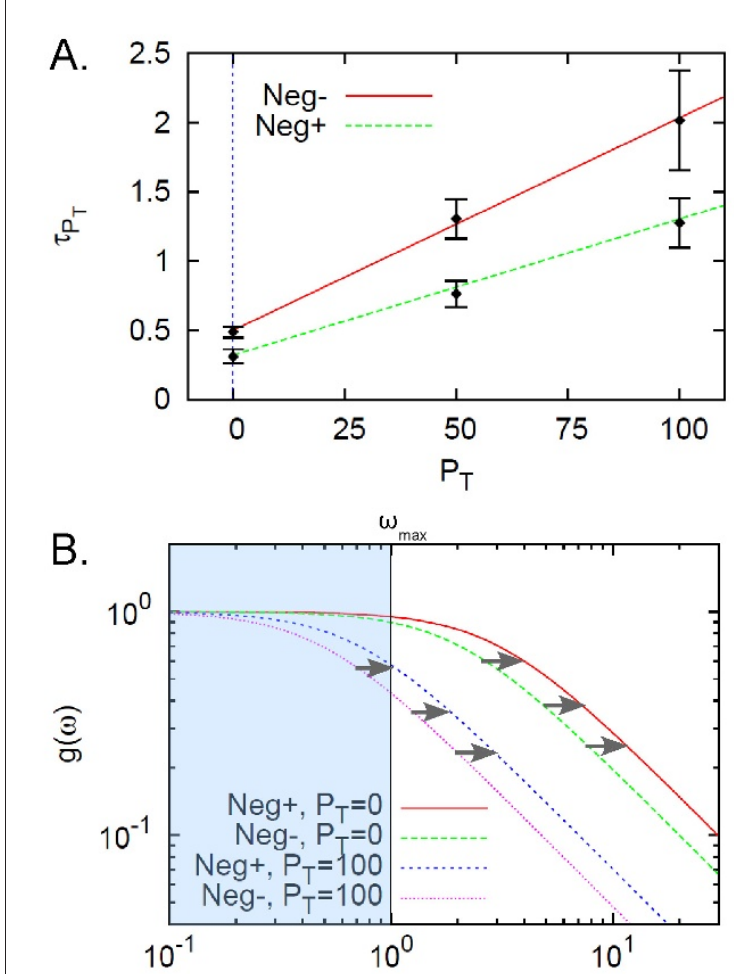

$\omega$

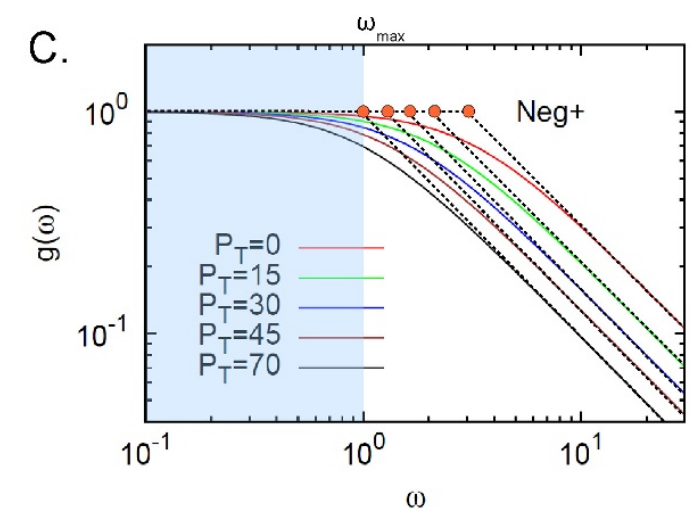

Figure 8 Estimation of fan-out from the measured response time constants. The module interface process involving dimer TFs is considered when the TF-expression is under inhibitory selfregulation (shown in Fig. 5A and reaction process (14)). (A) The response time constant $\tau_{P_{T}}$ of the transcription factor increases linearly with the number of the downstream promoters $P_{T}$. The time constants were measured from the autocorrelation functions of the concentration levels of the total transcription factors $(Y=X+$ $\left.2 X_{2}+2 P_{b}\right)$. The measured time constants are consistent with the deterministic prediction (lines) and are used to obtain the slope and y-intercept that are $R C_{1}$ and $R C\left(=\tau_{0}\right)$, respectively, by performing a linear fit. From the obtained $R C_{1}$ and $R C$, the fan-out function is estimated. (B) The normalized signal gain of the output signal $Y$ with respect to the input signal $\alpha$ (translation rate) is plotted for different frequencies. With negative feedback, the bandwidth increases. (C) The increased bandwidth results in the fan-out enhancement.

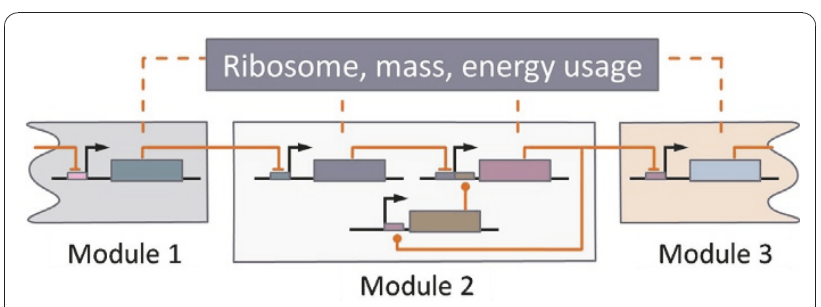

Figure 9 Effect of cell's machinery on fan-out. The fan-out of Module 1's output can be affected by downstream Modules 2 and 3 via the cell's machinery (ribosomes, mass and energy usage).

the input (translation rate) was plotted for different frequencies as shown in Figure 8B and 8C, and the cut-off frequency was shown to increase due to the negative feedback and to push the cut-off frequency away from the maximum desired operating frequency. This allows for a larger load from the downstream, resulting in the enhanced fan-out.

\section{Effect of cell's machinery on fan-out}

What we have not considered in this paper is the important case of cascading gene regulatory circuits (Figure 9). The question that arises here is whether the fan-out and retroactivity is affected in these situations. The answer depends on whether the transcription factors impose a significant load on the cell's machinery (ribosomes, mass and energy usage) or not. If not, then the fan-out values are unaffected because there is no upstream information transmission from downstream transcription factors. However, if the load on the cellular machinery is considered, then implied feedback and feed-forward loops appear, and this will have an effect on both the individual and net fan-out measures (Figure 9). This important topic is, however, beyond the scope of this paper and will be considered in a later publication.

\section{Conclusions}

In this paper, the concept and quantitative measure of fan-out have been introduced for genetic circuits. The fan-out is a measure of the maximum number of promoter sites that the output TFs of the upstream module can regulate without significant slow-down in the kinetics of the output. In addition, an efficient experimental method to estimate the fan-out have been proposed. The fan-out has been shown to be enhanced by self-inhibitory regulation on the output. In the estimation process of the fan-out, the retroactivity can also be computed. This study provides a way for quantifying the level of modularity in gene regulatory circuits and helps characterize and design module interfaces and therefore the modular construction of gene circuits. 


\section{Methods}

This section shows the mathematical derivations of the time-constant $\tau_{P_{T}}$ and fan-out functions for various cases.

\section{Monomer TF}

Consider the simple MIP shown in Figure 3. Under the quasi-equilibrium in the binding-unbinding process, the concentration of the bound TF is obtained as $P_{b}=P_{T} X /$ $\left(X+K_{d}\right)$. This equation is simplified to, by introducing $f$ $(X) \equiv X /\left(X+K_{d}\right)$

$$
P_{b}=P_{T} f(X)
$$

The time evolution of the total transcription factor ( $Y$ $\left.=X+P_{b}\right)$ is governed by the following equation [43]:

$$
\frac{d Y}{d t}=\alpha-\gamma X
$$

The response time constant of $Y$ is derived rather than that of $X$, because $Y$ is a pure slow variable showing the dynamics of our interest $[17,33,43]$. The response time constant of $Y$, denoted by $\tau_{P_{T}}$, is obtained by taking the derivative on the right hand side of the above equation with respect to $Y$ :

$$
\tau_{P_{T}}=-\left[\frac{d(\alpha-\gamma X)}{d Y}\right]^{-1}=\tau_{0} \frac{d Y}{d X}
$$

with $\tau_{0} \equiv \gamma^{-1}$. By using $Y=X+P_{b}$, the above equation becomes $\tau_{P_{T}}=\tau_{0}\left[1+\frac{d P_{b}}{d X}\right]$. By using Eq. (15), the time constant is obtained: $\tau_{P_{T}}=\tau_{0}\left(1+f^{\prime}(X) P_{T}\right)$. By comparing this with Eq. (10), $C / C_{1}$ becomes $K_{d}(1+X /$ $\left.K_{d}\right)^{2}$.

\section{Oligomer TF under directed degradation and self- regulation}

Consider that the transcription factors are composed of $n$ monomers described in Figure $5 \mathrm{~A}$. The bindingunbinding process is assumed to be in equilibrium, and $P_{b}$ is obtained as

$$
P_{b}=\frac{P_{T} X^{n}}{X^{n}+K_{d}} \equiv P_{T} f(X)
$$

The time evolution of $Y\left(=X+n P_{b}\right)$ is governed by

$$
\frac{d Y}{d t}=v_{1}(X)-v_{2}(X)
$$

The response time constant of $Y$ is obtained as

$$
\tau_{P_{T}}=-\left[\frac{d\left[v_{1}(X)-v_{2}(X)\right]}{d Y}\right]^{-1}=\tau_{0} \frac{d Y}{d X}
$$

where $\tau_{0} \equiv 1 /\left(\varepsilon_{2}-\varepsilon_{1}\right)$ with $\varepsilon_{1} \equiv \partial \nu_{1} / \partial X$ and $\varepsilon_{2} \equiv d \nu_{2}$ $(X) / d X$. By using $Y=X+n P_{b}$, the response time is obtained as

$$
\tau_{P_{T}}=\tau_{0}\left[1+P_{T} n \frac{d f(X)}{d X}\right]
$$

Where $n \frac{d f(X)}{d X}$ is defined to be $C_{1} / C$ by comparing the above equation with Eq. (10).

\section{Multiple promoters having different affinities}

We consider the case that two different types of TF-specific promoter plasmids, having different affinities for the TF and different strength of the origin of replication. The TF is assumed to be a monomer. The concentration of the TF bound on the promoter of each type $(i)$ is given as $P_{b i}=\frac{P_{T i} X}{X+K_{d i}} \equiv P_{T i} f_{i}(X)$ for $i=1,2$. The response time constant $\tau_{P_{T 1}, P_{T 2}}$ is given by Eq. (16). By using $Y=X+P_{b 1}+P_{b 2}, \tau_{P_{T 1}, P_{T 2}}$ becomes $\tau_{0}\left[1+\frac{d P_{b 1}}{d X}+\frac{d P_{b 2}}{d X}\right]$ which is rewritten as $\tau_{P_{T 1}, P_{T 2}}=\tau_{0}\left[1+f_{1}^{\prime}(X) P_{T 1}+f_{2}^{\prime}(X) P_{T 2}\right]$ by using $P_{b i}$ $=P_{T i} f_{i}(X)$. Finally by equating $\tau_{P_{T 1}, P_{T 2}}$ to $1 / \omega_{\max }$, Eq. (12) is obtained. If there were $n$-different types of promoter plasmids, the above equation is changed by replacing the last two terms to the sum over all the $n$-types.

In the RC-circuit representation, $\tau_{P_{T 1}, P_{T 2}}$ is given by $R C_{T}$ and the total capacitance $C_{T}$ is obtained as $C_{T}=C$ $+C_{1} P_{T 1}+C_{2} P_{T 2}$ with $C_{i}=\tau_{0} f_{i}^{\prime}(X) / R$. This indicates that this MIP can be mapped to an $\mathrm{RC}$-circuit having two different capacitances connected in parallel to $C$ as shown in Figure 5B.

\section{Software}

All stochastic simulations were carried out using our own Gillespie code written in C and run on a Quadcore PC under the Ubuntu Linux OS. Certain frequency plots, deterministic simulations and SBML translation of models to Jarnac script $[46,47]$ were carried out using SBW $[48,49]$. 


\section{Additional material}

Additional file 1: Supplementary document. Description of the repressilator BioModel, and mathematical derivation of a linear relationship between $C^{\prime}$ and $P_{T}$ for individual cases of oligomer TFs (with and without negative feedback) and degradation-tagged TFs.

Additional file 2: Mathematica notebook. Computation of fan-out values and frequency responses for the case of oligomer TFs that are under inhibitory self-regulation.

\section{Acknowledgements}

This work was supported by a National Science Foundation (NSF) Grant in Theoretical Biology 0827592. Preliminary studies were supported by funds from NSF FIBR 0527023. The authors acknowledge useful discussions with Hong Qian and Suk-jin Yoon.

\section{Authors' contributions}

KHK primarily developed and carried out the proposed work. HS participated its design and provided critical feedback and suggestions. All authors read and approved the final manuscript.

\section{Competing interests}

The authors declare that they have no competing interests.

Received: 11 September 2010 Accepted: 17 December 2010 Published: 17 December 2010

\section{References}

1. Sauro HM: Modularity defined. Mol Syst Bio/ 2008, 4:166.

2. Ayers JE: Digital integrated circuits: analysis and design Boca Raton: CRC Press; 2004.

3. Alon U: Network motifs: theory and experimental approaches. Nat Rev Genet 2007, 8(6):450-461.

4. Purnick PEM, Weiss R: The second wave of synthetic biology: from modules to systems. Nat Rev Mol Cell Biol 2009, 10(6):410-422.

5. Lucks JB, Qi L, Whitaker WR, Arkin AP: Toward scalable parts families for predictable design of biological circuits. Curr Opin Microbiol 2008, 11(6):567-573.

6. Keasling JD: Synthetic biology for synthetic chemistry. ACS Chem Biol 2008, 3:64-76.

7. Entus R, Aufderheide B, Sauro HM: Design and implementation of three incoherent feed-forward motif based biological concentration sensors. Syst Synth Biol 2007, 1:119-128.

8. Voigt CA: Genetic parts to program bacteria. Curr Opin Biotechnol 2006, 17(5):548-557.

9. Sprinzak D, Elowitz MB: Reconstruction of genetic circuits. Nature 2005, 438(7067):443-448

10. Endy D: Foundations for engineering biology. Nature 2005, 438(7067):449-453.

11. Hasty J, McMillen D, Collins JJ: Engineered gene circuits. Nature 2002, 420(6912):224-230

12. Stelling J, Sauer U, Szallasi Z, Doyle FJ III, Doyle J: Robustness of cellular functions. Cell 2004, 118(6):675-685.

13. Sauro HM, Kholodenko BN: Quantitative analysis of signaling networks. Prog Biophys Mol Biol 2004, 86:5-43.

14. Bergmann F, Vallabhajosyula R, Sauro H: Computational tools for modeling protein networks. Current Proteomics 2006, 3(3):181-197.

15. Sauro HM, Ingalls B: MAPK cascades as feedback amplifiers. arXiv:0710.5195[q-bio.MN] 2007.

16. Saez-Rodriguez J, Kremling A, Gilles ED: Dissecting the puzzle of life: modularization of signal transduction networks. Comput Chem Eng 2005, 29(3):619-629.

17. Del Vecchio D, Ninfa AJ, Sontag ED: Modular cell biology: retroactivity and insulation. Mol Syst Biol 2008, 4:161.

18. Lancaster DE: TTL Cookbook Indianapolis: H.W. Sams; 1974

19. Jayanthi S, Vecchio DD: On the Compromise between Retroactivity Attenuation and Noise Amplification in Gene Regulatory Networks.
Proceedings of the 48th IEEE Conference on Decision and Control: 15-18 December 2009; Shanghai, China 2010, 4565-4571.

20. Anishchenko VS, Astakhov V, Neiman A, Vadivasova T, Schimansky-Geier L: Nonlinear dynamics of chaotic and stochastic systems Berlin; New York: Springer-Verlag; 2002.

21. Simpson ML, Cox CD, Sayler GS: Frequency domain analysis of noise in autoregulated gene circuits. Proc Natl Acad Sci USA 2003, 100(8):4551.

22. Rosenfeld N, Young JW, Alon U, Swain PS, Elowitz MB: Gene regulation at the single-cell level. Science 2005, 307(5717):1962-1965.

23. Austin DW, Allen MS, McCollum JM, Dar RD, Wilgus JR, Sayler GS, Samatova NF, Cox CD, Simpson ML: Gene network shaping of inherent noise spectra. Nature 2006, 439:608-611.

24. Weinberger LS, Dar RD, Simpson ML: Transient-mediated fate determination in a transcriptional circuit of HIV. Nat Genet 2008, 40(4):466-470.

25. Dunlop MJ, Cox RS III, Levine JH, Murray RM, Elowitz MB: Regulatory activity revealed by dynamic correlations in gene expression noise. Nat Genet 2008, 40(12):1493-1498.

26. Rao CV, Wolf DM, Arkin AP: Control, exploitation and tolerance of intracellular noise. Nature 2002, 420(6912):231-237.

27. Raser JM, O'Shea EK: Control of stochasticity in eukaryotic gene expression. Science 2004, 304(5678):1811-1814

28. Kærn M, Elston TC, Blake WJ, Collins JJ: Stochasticity in gene expression: from theories to phenotypes. Nat Rev Genet 2005, 6(6):451-464.

29. Shahrezaei V, Ollivier JF, Swain PS: Colored extrinsic fluctuations and stochastic gene expression. Mol Syst Biol 2008, 4:196.

30. Elowitz MB, Leibler S: A synthetic oscillatory network of transcriptional regulators. Nature 2000, 403(6767):335-338.

31. Nilsson J, Riedel S: Electric circuits Upper Saddle River, N.J.: Pearson Prentice Hall; 2008 .

32. Kepler TB, Elston TC: Stochasticity in transcriptional regulation: origins, consequences, and mathematical representations. Biophys J 2001, 81(6):3116-3136

33. Rao CV, Arkin AP: Stochastic chemical kinetics and the quasi-steady-state assumption: Application to the Gillespie algorithm. J Chem Phys 2003, 118:4999.

34. Simpson ML, Cox CD, Sayler GS: Frequency domain chemical Langevin analysis of stochasticity in gene transcriptional regulation. J Theor Biol 2004, 229(3):383-394

35. Buchler NE, Cross FR: Protein sequestration generates a flexible ultrasensitive response in a genetic network. Mol Syst Biol 2009, 5:272

36. Fell DA: Metabolic control analysis: a survey of its theoretical and experimental development. Biochem J 1992, 286:313-330.

37. Shen-Orr SS, Milo R, Mangan S, Alon U: Network motifs in the transcriptional regulation network of Escherichia coli. Nature Genet 2002, 31:64-68.

38. Yu R, Pesce C, Colman-Lerner A, Lok L, Pincus D, Serra E, Holl M, Benjamin K, Gordon A, Brent R: Negative feedback that improves information transmission in yeast signalling. Nature 2008, 456(7223):755-761.

39. Arkin A, Ross J, McAdams HH: Stochastic kinetic analysis of developmental pathway bifurcation in phage lambda-infected Escherichia coli cells. Genetics 1998, 149(4):1633-1648.

40. Elowitz MB, Levine AJ, Siggia ED, Swain PS: Stochastic gene expression in a single cell. Science 2002, 297(5584):1183-1186

41. Ozbudak EM, Thattai M, Kurtser I, Grossman AD, van Oudenaarden A: Regulation of noise in the expression of a single gene. Nat Genet 2002, 31:69-73.

42. Elf J, Li GW, Xie XS: Probing transcription factor dynamics at the singlemolecule level in a living cell. Science 2007, 316(5828):1191-1194.

43. Kim KH, Sauro HM: Measuring the degree of modularity from gene expression noise in gene regulatory circuits. Biophys $J 2011$ arXiv:0910.5522[q-bio.MN] 2009

44. Gillespie DT: Exact stochastic simulation of coupled chemical reactions. J Phys Chem 1977, 81:2340-2361.

45. Wolfram S: Mathematica: a system for doing mathematics by computer Redwood City, CA: Addison-Wesley Pub. Co., Advanced Book Program 1988

46. Sauro H: Jarnac: A system for interactive metabolic analysis. Animating the Cellular Map: Proceedings of the 9th International Meeting on BioThermoKinetics 2000, 221-228. 
47. Smith L, Bergmann F, Chandran D, Sauro H: Antimony: a modular model definition language. Bioinformatics 2009, 25(18):2452.

48. Sauro H, Hucka M, Finney A, Wellock C, Bolouri H, Doyle J, Kitano H: Next generation simulation tools: the Systems Biology Workbench and BioSPICE integration. Omics A Journal of Integrative Biology 2003, 7(4):355-372.

49. Bergmann F, Sauro H: SBW-a modular framework for systems biology. Proceedings of the 38th conference on Winter simulation: 3-6 December 2006; Monterey, CA 2006, 1637-1645.

50. Le Novère $N$, Bornstein $B$, Broicher $A$, Courtot $M$, Donizelli $M$, Dharuri $H$, Li L, Sauro H, Schilstra M, Shapiro B, Snoep JL, Hucka M: BioModels Database: a free, centralized database of curated, published, quantitative kinetic models of biochemical and cellular systems. Nucleic Acids Res 2006, , 34 Database: D689-D691.

doi:10.1186/1754-1611-4-16

Cite this article as: Kim and Sauro: Fan-out in gene regulatory networks. Journal of Biological Engineering 2010 4:16.

\section{Submit your next manuscript to BioMed Central} and take full advantage of:

- Convenient online submission

- Thorough peer review

- No space constraints or color figure charges

- Immediate publication on acceptance

- Inclusion in PubMed, CAS, Scopus and Google Scholar

- Research which is freely available for redistribution

Submit your manuscript at www.biomedcentral.com/submit 